\title{
Health Behavior in School-Aged Children: Summary of the 2013/2014 WHO Survey
}

Babitha Shanmuganandapala, BScN, RN

York University

The following piece summarizes the key findings of the 2013/2014 Health Behavior in School-Aged Children report 'Growing up unequal: gender and socioeconomic differences in young people's health and well-being'.

Youth between the ages of 11 and 15 face unique stresses and challenges such as mounting academic expectations, shifting peer and family relationships, and the emotional and physical transformations associated with the transition into adolescence. During this transition period, youth start developing and exercising the ability to making independent decisions that may influence their health and health related behavior. Behaviours developed at this critical stage of the life-course can continue into adulthood and affect multiple aspects of their health.

For over 30 years, the WHO has examined and delivered data on 11-, 13- and 15 year old boys' and girls' health, well-being, social environment, and health behavior through the collaborative cross-national study Health Behavior in School-Aged Children (HBSC). Using random sampling, data are collected in all participating countries and regions through school-based, self-report surveys. By studying young people's health in their social context, researchers hope to understand how these aspects impact young people's health during their transition into adolescence. HBSC uses study results at nationwide and worldwide levels to obtain new insight into young people's health and wellbeing, understand the social determinants of health, and inform policy and practice to improve young people's lives.

The most recent international HBSC report 'Growing up unequal: gender and socioeconomic differences in young people's health and well-being' offers findings from the 2013/2014 survey, on the health of approximately 220,000 young people from 42 countries in Europe and North America. Participating countries included Finland, Norway, Austria, Belgium, Hungary, Israel, Scotland, Spain, Sweden, Switzerland, Wales, Denmark, Canada, Latvia, Poland, Belgium, Czech Republic, Estonia, France, Germany, Greenland, Lithuania, Russian Federation, Slovakia, England, Greece, Portugal, Ireland, the former Yugoslav Republic of Macedonia, Netherlands, Italy, Croatia, Malta, Slovenia, Ukraine, Iceland, Luxembourg, Romania, Armenia, Bulgaria, Albania and Republic of Moldova. This report places a special emphasis on how gender and socioeconomic differences affect the way that youth mature and develop. Topics covered by the survey included social context (relationships with family, peers and school), health outcomes (perceived health, mental health, injuries, and topics related to body weight), health behavior (eating habits, oral health and physical activity), and risk behaviors (use of alcohol, tobacco and cannabis, sexual behavior, fighting and bullying). Family and peer support, migration, cyber-bullying and serious injuries are new topics of focus included in the report.

The report indicates that the protective role of a family actually disappears during this life stage, particularly for girls as perceived support from friends stays constant. In terms of interactions and communication related to technological advances, youth participating in everyday electronic media communication with peers proves to be an increasing trend; however, large variations exist between countries/regions, emphasizing possible cultural factors also playing a role. Data related to cyber-bullying revealed that this form of bullying remains less common than traditional bullying. There also appeared to be significant differences in youth's experiences at school, specifically concerning how much they like school and feel pressured by school work. This may be due to the different school systems in varying regions; however, younger boys and older girls are noted to be more likely to experience this type of stress.

Findings on girls also indicate a marked decline in subjective well-being during the years of adolescence. In addition to school stress being a possible factor in influencing lower levels of mental well-being, girls' body dissatisfaction was also found to increase significantly during this period. This trend was especially noted in Western and central European countries, even though actual weights remained stable. The study found that on average, one in five girls 
reported fair or poor health by age 15 with half of them experiencing multiple health complaints more than once a week. Additionally, girls report the lowest levels of life satisfaction, daily breakfast consumption and physical activity. However, in terms of positive behaviors, girls are more likely to include fruit and vegetables in their diet and brush their teeth, while boys are more likely to be physically active. Other gendered findings include boys being more likely to experience injury, be involved in physical fights, drink alcohol and smoke tobacco more often. These results may seem discouraging; however, when compared to previous surveys, the findings indicate a significant overall decline in substance use, fighting, and bullying victimization among boys and girls in many countries and regions.

Findings related to family affluence show that youth from "low-affluence families tend to have poorer health, lower life satisfaction, higher levels of obesity and sedentary behaviors, poorer communication with their parents, less social interaction via social media and lower levels of support from friends and family." (WHO, 2016, pg.233). Better outcomes were reported by youth from high-affluence families. The relationship between family affluence is not as strong for risk behaviors and school experience which may indicate that irrespective of family socioeconomic status, school environments can be of support to youth's health and development. It is of importance to note that health related behaviors are also affected by structural determinants of health- the social and economic systems of each country as well as social and cultural norms.

Some of the policy recommendations include: the usage of new communication technologies for health related information dissemination; prevention programs early and with a "gendered lens" for gender identified issues; integrating oral health promotion with general health promotion; increasing access to sexual health services and contraceptives; using a Health in All Policies approach to address subjective mental health; interventions to prevent drug experimentation, adoption and access; systemic approach to address obesity through school food environment, provision of healthier foods, safe neighborhoods and opportunities for physical activity; injury prevention through use of legislation, product/environmental changes, promotion of safety devices, and education; support the establishment and maintenance of social relationships by increasing such opportunities i.e. for peer interaction in safe and structured settings; and lastly, more programs that promote positive parenting.

In the ever changing global context, identifying youth related changes, trends and patterns are essential to recognizing, assessing, and addressing their physical and mental health needs. Healthy development during this crucial life stage can prevent health problems and translate into lifelong positive health habits. By recognizing the intersections of age, gender, class, social context within the individual, familial, community, and societal levels, we can increase the understanding of, and opportunities for healthcare and social systems to shape health promotion, public policies, practice/service delivery, and enhance further research for, and within, this population. The WHO's HBSC report is a crucial and necessary tool for such positive actions worldwide.

\section{Reference:}

World Health Organization. (2016). Growing up unequal: Gender and socioeconomic differences in young people's health and well-being. Retrieved from http://www.euro.who.int/ data/assets/pdf file/0003/303438/HSBC-No7Growing-up-unequal-full-report.pdf?ua=1 